\title{
Skill sharing in a pandemic: five minutes with . . . Helgi Johannsson
}

\author{
The consultant anaesthetist and Royal College of Anaesthetists council member discusses supporting \\ anaesthetists to work in intensive care during the covid-19 pandemic
}

\section{Abi Rimmer}

The BMJ

"At the moment, demand for intensive care capacity massively outstrips supply. If we left intensive care doctors to look after these patients by themselves they just wouldn't be able to cope. "Anaesthesia and intensive care are similar specialties in many ways. All anaesthetists have to do a fair amount of intensive care in their training and all intensive care consultants have to do some anaesthesia in their training.

"Where we differ is that most of anaesthesia's bread and butter is doing operations while intensive care is looking after patients. But anaesthetists also look after patients who need operations. It's not that big a leap for us to go back into intensive care; so that's what we've done.

"In my hospital we've cancelled all elective surgery and the anaesthetists who would normally be doing that have been drafted in. We've set up a full shift system covering a new intensive care unit that didn't exist before. Crucially we're also still covering emergency anaesthesia, obstetrics, and other emergencies that still come through.

"As preparation, most of us have gone on the intensive care ward rounds. A lot of it is just about learning the protocols and the policies of the unit and making sure we're familiar with how those get done. For example, feeding protocols-obviously, during an operation we don't feed our patients, so it's all those little areas that we may not have thought about which become important when we're moving across.

"I know that some of our trainees who weren't in an anaesthesia or an intensive care specialty were anxious about starting in intensive care but hopefully we've made them feel at home and supported them. It's important that we don't just dump people in the middle of a covid-19 unit without training them properly. "It's about making sure they know what they're expected to do and, crucially, what they are not expected to do and when to call for help. We don't want them to feel out of their depth.

"I've also learnt from the past few days that it's actually quite hard wearing all the protective equipment and trying to do a clinical job where communication is important. With a facemask and a visor on, nobody being able to see what expression you are making, and your glasses steaming up—that's all quite difficult.

"While I hope we don't have to, we're preparing to bring in other specialties that aren't so closely aligned with intensive care, such as surgeons. Surgeons are highly trained and they're used to looking after postoperative patients, many of whom will get sepsis and behave in a similar way to patients with covid-19-there's a definite overlap in their skill set. They are, however, not familiar with ventilators so it takes a bit longer to bring their ventilator skills up to the required level.

"Overall I've been really impressed with the flexibility and the team spirit of everyone. Not just the doctors but the nurses as well. At the moment we have a mix of intensive care and non-intensive care nurses on our ward and the non-intensive care nurses are doing an amazing job. Everyone is just trying to cope with what is a really difficult time." 